\title{
APPLICATIONS AND GENERALIZATIONS OF THE CONCEPTION OF
}

\section{ADJOINT SYSTEMS*}

\author{
BY \\ MAXIME BÔCHER
}

The highly fruitful conception of adjoint pairs of linear systems, each consisting of a homogeneous linear differential equation of the $n$th order and of $n$ homogeneous linear boundary conditions, was first introduced in an explicit and general manner by BiRKHOFF. $\dagger$ That we have here a conception of fundamental importance is hardly yet generally appreciated. It is my purpose in the present paper to make an application of this idea to an important question in the theory of linear boundary problems which has so far been treated only in very special cases. $\ddagger$ I will mention in passing that the results may also be obtained by the use of integral equations; though, if we wish to include all cases, not perhaps so simply or obviously as might at first sight appear.

In $\S 1$ the conception of adjoint system and a few other fundamental matters concerning linear systems are explained and a few preliminary questions disposed of. In $\S 2$ come the main results of the paper, namely Theorems I and II, which apply to the case of complex as well as to that of real independent variable. These theorems are then applied in some detail to the equation of the second order, whereby the relation to MASON's results becomes apparent. In $\S 3$ an extension to systems of equations of any order is briefly indicated; and a somewhat generalized type of differential equation is also considered. Finally, in $\S 4$, the conception of adjoint system is extended to the case in which the number of boundary conditions is not the same as the order of the differential equation.

\section{§1. General Preliminaries.}

In the differential expression

$$
L(u)=l_{n} \frac{d^{n} u}{d x^{n}}+l_{n-1} \frac{d^{n-1} u}{d x^{n-1}}+\cdots+l_{0} u
$$

* Presented to the Society April 26, 1913. Section 4 has been added since.

† These Transactions, vol. 9 (1908), p. 373.

f Cf. Mason, these T r a n a a c t i o n s, vol. 7 (1906), pp. 340-344, where the self-adjoint system of the second order is treated. 
we assume that $l_{n}$ does not vanish at any point of a certain finite region $S$ which may be either a simply connected two-dimensional continuum in the complex $x$-plane, or any connected piece of the $x$-axis. In the former case we also assume that all the coefficients $l_{i}$ are analytic functions of $x$ throughout $S$; in the latter, that they are continuous but not necessarily real functions of the real variable $x$, and that $l_{i}$ has continuous derivatives of the first $i$ orders.*

This expression $L$ is connected with its adjoint

$$
M(v)=(-1)^{n} \frac{d^{n}\left(l_{n} v\right)}{d x^{n}}+(-1)^{n-1} \frac{d^{n-1}\left(l_{n-1} v\right)}{d x^{n-1}}+\cdots+l_{0} v
$$

by Lagrange's identity

$$
v L(u)-u M(v)=\frac{d}{d x} P(u, v),
$$

where $P$ is a bilinear form in $u, u^{\prime}, \cdots u^{[n-1]}, v, v^{\prime}, \cdots v^{[n-1]}$. This bilinear form is non-singular for every value of $x$ in $S$ since in its matrix the elements in the secondary diagonal are alternately $l_{n}$ and $-l_{n}$, while all the elements below this diagonal are zero. $\dagger$

If we integrate this identity from $a$ to $b$, these being any two points in $S$, we get Green's theorem, the second member of which is therefore a nonsingular bilinear form in the quantities

$$
\begin{aligned}
& u(a), u^{\prime}(a), \cdots u^{[n-1]}(a), u(b), u^{\prime}(b), \cdots u^{[n-1]}(b), \\
& v(a), v^{\prime}(a), \cdots v^{[n-1]}(a), v(b), v^{\prime}(b), \cdots v^{[n-1]}(b) .
\end{aligned}
$$

Let $U_{1}(u), \cdots U_{2 n}(u)$ be any $2 n$ linearly independent linear forms in (1). The quantities (1) can then be expressed linearly in terms of $U_{1}, \cdots U_{2 n}$. The bilinear form in the second member of Green's theorem thus becomes a linear form in the $U$ 's whose coefficients, which we denote by $V_{2 n}(v), \cdots$ $V_{1}(v)$, are linear forms in (2). Thus Green's theorem takes the form

$$
\begin{aligned}
\int_{a}^{\iota}[v L(u)-u M(v)] d x=U_{1}(u) V_{2 n}(v) & +U_{2}(u) V_{2 n-1}(v) \\
& +\cdots+U_{2 n}(u) V_{1}(v) .
\end{aligned}
$$

* This restriction on the derivatives of the functions $l_{i}$ is a sufficient but not a necessary one to secure the existence of an adjoint, that is of an expression $M(v)$ satisfying Lagrange's identity. For instance, if $n=2$, it is sufficient to demand that $l_{2}$ have a continuous derivative $l_{2}^{\prime}$, and that $l_{2}^{\prime}-l_{1}$ also have a continuous derivative. The adjoint is then

$$
M(v)=l_{2} \frac{d^{2} v}{d x^{2}}+\left(2 l_{2}^{\prime}-l_{1}\right) \frac{d v}{d x}+\left[\frac{d}{d x}\left(l_{2}^{\prime}-l_{1}\right)+l_{0}\right] v .
$$

For still further generalization see the closing paragraph of $\S 3$.

† This may be seen by a reference to the explicit form for $P$ as given, for instance, in DARBoux's Theorie des Surfaces, vol. 2, p. 100. It may also be readily inferred from Lagrange's identity itself if we merely admit the general form of this identity. 
Moreover, since the bilinear form was non-singular, the $V$ 's must be linearly independent.

Now consider the linear system

$$
\begin{aligned}
L(u) & =r, \\
U_{i}(u) & =\gamma_{i} \quad(i=1,2, \cdots n),
\end{aligned}
$$

where $L$ has the same meaning as before, $r$ is a function of $x$ continuous in $S$ (and, if $S$ is a two dimensional continuum in the complex plane, analytic in $S$ ), the $\gamma_{i}$ 's are constants, and the $U_{i}$ 's are linear forms in the quantities (1) which are restricted merely by being assumed linearly independent.

By the reduced system of (4), (5) we understand the homogeneous system

$$
\begin{aligned}
L(u) & =0, \\
U_{i}(u) & =0 \quad(i=1,2, \cdots n) .
\end{aligned}
$$

Now form $n$ further linear forms $U_{n+1}(u), \cdots U_{2 n}(u)$ of the quantities (1) subject merely to the condition that $U_{1}, \cdots U_{2 n}$ are linearly independent; and, by means of them, form, as above, the linear forms $V_{1}, \cdots V_{2 n}$. The system

$$
\begin{aligned}
M(v) & =0, \\
V_{i}(v) & =0
\end{aligned}
$$$$
(i=1,2, \cdots n)
$$

is called a system adjoint to (6), (7). It is clear from the symmetry of the formulæ that, conversely, (6), (7) is a system adjoint to (8), (9)*.

A homogeneous system like (6), (7) is called compatible or incompatible according as it has or has not a solution other than zero. It is said to have $k$-fold compatibility if it has $k$ and only $k$ linearly independent solutions; and zero-fold compatibility shall be understood to mean incompatibility. If we indicate by $y_{1}, \cdots y_{n}$ linearly independent solutions of (6), we establish, by assuming the general solution of (6) in the form $c_{1} y_{1}+\cdots+c_{n} y_{n}$ and determining the $c$ 's so that this expression satisfies (7) also,

Lemma I. $\dagger$ A necessary and sufficient condition that the system (6),

* It may be seen by very simple algebraic considerations that if we make a different choice of the arbitrary expressions $U_{n+1}, \cdots U_{2 n}$, we shall obtain an adjoint system essentially identical with the one already found, the only difference being that the conditions (9) are replaced by homogeneous linear combinations of themselves with non-vanishing determinant, and that $V_{n+1}, \cdots V_{2 n}$ are changed only by having homogeneous linear combinations of $V_{1}, \cdots V_{n}$ added to them. These facts are not, however, necessary for what follows.

$\dagger$ Given substantially by MAsON and BirkноFr, loc. cit. 
(7) have $k$-fold compatibility is that the matrix

$$
\left(\begin{array}{ccc}
U_{1}\left(y_{1}\right) & \cdots & U_{1}\left(y_{n}\right) \\
\cdots & \cdots & \cdots \\
\cdots & \cdots & \cdots \\
U_{n}\left(y_{1}\right) & \cdots & U_{n}\left(y_{n}\right)
\end{array}\right)
$$

be of rank $n-k$, where $y_{1}, \cdots y_{n}$ is a fundamental system of (6).

Similarly, if we denote by $u_{0}$ a particular solution of (4), so that the general solution is $u_{0}+c_{1} y_{1}+\cdots+c_{n} y_{n}$, and if we determine these $c$ 's in such a way that this function satisfies (5) also, we find

Lemma II. If the reduced system (6), (7) has $k$-fold compatibility, a necessary and sufficient condition that the complete system (4), (5) have a solution is that the matrix

$$
\left(\begin{array}{cccc}
U_{1}\left(y_{1}\right) & \cdots & U_{1}\left(y_{n}\right) & U_{1}\left(u_{0}\right)-\gamma_{1} \\
\cdots & \cdots & \cdots & \cdots \\
\cdots & \cdots & \cdots & \cdots \\
U_{n}\left(y_{1}\right) & \cdots & U_{n}\left(y_{n}\right) & U_{n}\left(u_{0}\right)-\gamma_{n}
\end{array}\right)
$$

be of rank $n-k$, where $y_{1}, \cdots y_{n}$ is a fundamental system of (6), and $u_{0}$ a particular solution of (4).

If $k=0$, we see from Lemma $I$ that the matrix last written is always of rank $n$. Hence

LEMma III.* If the reduced system (6), (7) is incompatible, the complete system (4), (5) always has a solution.

We add finally

LEMmA IV. If $u_{1}, \cdots u_{k}$ are linearly independent solutions of the homogeneous system (6), (7), then the $k$ sets of constants

are linearly independent.

$$
U_{n+1}\left(u_{i}\right), \cdots U_{2 n}\left(u_{i}\right) \quad(i=1,2, \cdots k)
$$

For otherwise there would exist $k$ constants, $c_{1}, \cdots c_{k}$, not all zero, such that all the quantities

$$
c_{1} U_{j}\left(u_{1}\right)+\cdots+c_{k} U_{j}\left(u_{k}\right) \quad(j=n+1, \cdots 2 n)
$$

vanish. Consequently if we let

$$
u=c_{1} u_{1}+\cdots+c_{k} u_{k},
$$

we see, on the one hand, that $u$ is not identically zero, and, on the other, that the equations

$$
U_{1}(u)=0, \cdots U_{2 n}(u)=0
$$

* Mason, loc. cit., and, for a simpler proof, Bócher, A n a ls of Math e mati cs, $2 d$ ser., vol. 13 (1911), p. 74. The proof here given is simpler than either of these. 
are satisfied. These being $2 n$ linearly independent, homogeneous, linear equations in the $2 n$ quantities (1), it follows that all the quantities (1) vanish; but this impossible since then $u$ would vanish identically.

\section{§2. Conditions for Existence of Solutions of Non-Homogeneous Systems.}

Tireorem I.* If a homogeneous system (6), (7) has $k$-fold compatibility, the adjoint system (8), (9) also has k-fold compatibility.

On account of the reciprocal relation between a system and its adjoint, it will clearly be sufficient if we prove that (8), (9) has at least $k$-fold compatibility. For this purpose let $u$ be any solution of the system (6), ( 7 ), and $z_{1}, \cdots z_{n}$, linearly independent solutions of the equation (8). From Green's theorem, (3), by substituting for $v$ in succession the functons $z_{1}, \cdots z_{n}$, we find the equations

$$
\begin{aligned}
& U_{2 n}(u) V_{1}\left(z_{1}\right)+\cdots+U_{n+1}(u) V_{n}\left(z_{1}\right)=0 \text {, } \\
& U_{2 n}(u) V_{1}\left(z_{n}\right)+\cdots+U_{n+1}(u) V_{n}\left(z_{n}\right)=0 \text {. }
\end{aligned}
$$

These equations will be satisfied, in particular, if we replace $u$ by any of the $k$ linearly independent solutions $u_{1}, \cdots u_{k}$ of the system (6), (7). Consequently, regarded as a system of linear algebraic equations for determining $U_{2 n}, \cdots U_{n+1}$, equations (11) have the $k$ solutions

$$
U_{2 n}\left(u_{i}\right), \cdots U_{n+1}\left(u_{i}\right) \quad(i=1,2, \cdots k) .
$$

These solutions, by Lemma IV, are linearly independent. Consequently (11), regarded as a system of equations in the $U$ 's, has at least $k$ linearly independent solutions. Hence, as we know from the theory of linear homogeneous algebraic equations, the matrix

$$
\left(\begin{array}{ccc}
V_{1}\left(z_{1}\right) & \cdots & V_{n}\left(z_{1}\right) \\
\cdots & \cdots & \cdots \\
\cdots & \cdots & \cdots \\
V_{1}\left(z_{n}\right) & \cdots & V_{n}\left(z_{n}\right)
\end{array}\right)
$$

is of rank $n-k$ at most. From this, by Lemma $\mathrm{I}$, we infer that the system (९), (9) has at least $k$-fold compatibility, as was to be proved.

We come now to our main result.

'Theorem II. A necessary and sufficient condition that the complete system (4), (5) have a solution is that every solution, $v$, of the homogeneous adjoint system (8), (9) satisfy the relation

$$
\int_{u}^{b} v r d x=\gamma_{1} V_{2 n}(v)+\cdots+\gamma_{n} V_{n+1}(v) .
$$

* A special case of this theorem was established by Birknoff, loc. cit. 
In proving this theorem we may assume that the system (8), (9) has $k$-fold compatibility, where $k \overline{>}$; for if $k=0$, the theorem to be proved follows at once from Lemma III and Theorem I.

If the system (4), (5) has a solution, and we substitute in Green's theorem, (3), for $u$ this solution, and for $v$ any solution of (8), (9), we see at once that the condition of Theorem II is a necessary condition. To prove it sufficient,* let $v$ be any solution of the system (8), (9), and let $u_{0}$ be any solution of equation (4). From (3) we then get the equation

$$
\int_{a}^{b} v r d x=U_{1}\left(u_{0}\right) V_{2 n}(v)+\cdots+U_{n}\left(u_{0}\right) V_{n+1}(v) .
$$

By subtracting (12) from this equation, we find

$$
\left(U_{1}\left(u_{0}\right)-\gamma_{1}\right) V_{2 n}(v)+\cdots+\left(U_{n}\left(u_{0}\right)-\gamma_{n}\right) V_{n+1}(v)=0 .
$$

Now let $y_{1}, \cdots y_{n}$ be linearly independent solutions of (6). Then from (3) we obtain the equations

$$
U_{1}\left(y_{j}\right) V_{2 n}(v)+\cdots+U_{n}\left(y_{j}\right) V_{n+1}(v)=0 \quad(j=1, \cdots n) .
$$

Equations (13) and (14) together form a system of $n+1$ linear homogeneous algebraic equations in $V_{2 n}(v), \cdots V_{n+1}(v)$, so that if we denote by $v_{1}$, $\cdots v_{k}$ a system of linearly independent solutions of the system (8), (9), these linear algebraic equations have the $k$ solutions

$$
V_{2 n}\left(v_{i}\right), \cdots V_{n+1}\left(v_{i}\right) \quad(i=1,2, \cdots k),
$$

which by Lemma IV are linearly independent. It follows from the theory of linear algebraic equations that the rank of the matrix of the system (13), (14) is at most $n-k$; and it cannot be of lower rank than this since the matrix (10) is of rank $n-k$. The matrix of the system (13), (14) being essentially the matrix of Lemma II, it follows that the system (4), (5) has a solution.

The condition of Theorem II is peculiarly simple in the important special case in which the $\gamma_{i}$ 's are all zero. If this is not so, we should be obliged to compute the quantities $V_{i}$ before applying it. This computation we will now carry through in the case of the equation of the second order, which, without loss of generality, we take in the self-adjoint form

$$
L(u) \equiv \frac{d}{d x}\left(K \frac{d u}{d x}\right)-G u=r,
$$

* This proof of sufficiency was worked out in the case $n=2$ by Mr. Joseph SLepian, a student in one of my courses at Harvard. 
where $K$ does not vanish in $S .^{*} \quad$ We then have, as may be easily verified

$$
P(u, v)=K\left(v \frac{d u}{d x}-u \frac{d v}{d x}\right)
$$

The two boundary conditions (5) we will now write

$$
\begin{aligned}
& a_{1} u(a)+a_{2} u(b)+a_{3} u^{\prime}(a)+a_{4} u^{\prime}(b)=A, \\
& b_{1} u(a)+b_{2} u(b)+b_{3} u^{\prime}(a)+b_{4} u^{\prime}(b)=B .
\end{aligned}
$$

Since the first members of these equations have been assumed to be linearly independent, not all the determinants

$$
d_{i j}=a_{i} b_{j}-a_{j} b_{i}
$$

will be zero. We will prove the following facts:

If in Theorem II the differential equation is (16) and the boundary conditions (17), the condition (12) may in general be written in any of the following forms:

$$
\begin{aligned}
& d_{12} \int_{a}^{b} v r d x=K(a)\left(A b_{2}-B a_{2}\right) v^{\prime}(a)+K(b)\left(A b_{1}-B a_{1}\right) v^{\prime}(b), \\
& d_{43} \int_{a}^{b} v r d x=K(a)\left(A b_{4}-B a_{4}\right) v(a)+K(b)\left(A b_{3}-B a_{3}\right) v(b), \\
& d_{23} \int_{a}^{b} v r d x=K(a)\left(A b_{2}-B a_{2}\right) v(a)-K(b)\left(A b_{3}-B a_{3}\right) v^{\prime}(b), \\
& d_{14} \int_{a}^{b} v r d x=K(a)\left(A b_{4}-B a_{4}\right) v^{\prime}(a)-K(b)\left(A b_{1}-B a_{1}\right) v(b), \\
& d_{13} \int_{a}^{b} v r d x=K(a)\left(A b_{1}-B a_{1}\right) v(a)+K(a)\left(A b_{3}-B a_{3}\right) v^{\prime}(a), \\
& d_{42} \int_{a}^{b} v r d x=K(b)\left(A b_{2}-B a_{2}\right) v(b)+K(b)\left(A b_{4}-B a_{4}\right) v^{\prime}(b) .
\end{aligned}
$$

Of these forms only those will be inadmissible for which the $d_{i j}$ which occurs as a factor of the first member is zero.

In all cases, a necessary and sufficient condition that the system (16), (17) be self-adjoint is

$$
d_{24} K(a)=d_{13} K(b) .
$$

* In order that, when $x$ is restricted to real values, this expression, $L(u)$, satisfy the conditions stated in the text at the beginning of $\& 1$, the function $K$ must have continuous first and second derivatives. It will satisfy the conditions referred to in the first footnote of $\& 1$ if $K$ ha a continuous first derivative. The mere continuity of $K, G$, and $r$ is, however, sufficient for our purposes as will be seen by a reference to the closing lines of $\$ 3$. 
Let us write for brevity

$$
\begin{array}{llll}
u(a)=u_{1}, & u(b)=u_{2}, & u^{\prime}(a)=u_{3}, & u^{\prime}(b)=u_{4}, \\
v(a)=v_{1}, & v(b)=v_{2}, & v^{\prime}(a)=v_{3}, & v^{\prime}(b)=v_{4} .
\end{array}
$$

The bilinear form in the second member of Green's theorem may now be written

$$
K(b)\left(v_{2} u_{4}-u_{2} v_{4}\right)-K(a)\left(v_{1} u_{3}-u_{1} v_{3}\right),
$$

and we have

$$
\begin{aligned}
& U_{1}(u)=a_{1} u_{1}+a_{2} u_{2}+a_{3} u_{3}+a_{4} u_{4}, \\
& U_{2}(u)=b_{1} u_{1}+b_{2} u_{2}+b_{3} u_{3}+b_{4} u_{4} .
\end{aligned}
$$

Let us now first assume $d_{12} \neq 0$. In this case we may let

$$
U_{3}=u_{3}, \quad U_{4}=u_{4},
$$

and we readily compute the following expressions for the $V$ 's:

$$
\begin{aligned}
& V_{1}=\frac{1}{d_{12}}\left[K(b) d_{12} v_{2}+K(a) d_{24} v_{3}+K(b) d_{14} v_{4}\right], \\
& V_{2}=\frac{1}{d_{12}}\left[-K(a) d_{12} v_{1}+K(a) d_{23} v_{3}+K(b) d_{13} v_{4}\right], \\
& V_{3}=\frac{1}{d_{12}}\left[-K(a) a_{2} v_{3}-K(b) a_{1} v_{4}\right], \\
& V_{4}=\frac{1}{d_{12}}\left[K(a) b_{2} v_{3}+K(b) b_{1} v_{4}\right] .
\end{aligned}
$$

By equating the first two of these expressions to zero we get the adjoint boundary conditions, and we readily see that these are linear combinations of the original conditions (17) when and only when (19) is fulfilled. Thus we have established in this case that (19) is a necessary and sufficient condition that the system be self-adjoint.

On the other hand, by substituting in the condition of Theorem II the values just obtained for $V_{3}, V_{4}$ and making the necessary changes in notation, we find that this condition reduces to the first of conditions (18).

Assume next that $d_{13} \neq 0$. Letting

we find

$$
U_{3}=u_{2}, \quad U_{4}=u_{4},
$$

$$
V_{1}=\frac{1}{d_{13}}\left[K(a) d_{14} v_{1}+K(b) d_{13} v_{2}+K(a) d_{34} v_{3}\right],
$$




$$
\begin{aligned}
& V_{2}=\frac{1}{d_{13}}\left[K(a) d_{12} v_{1}-K(a) d_{23} v_{3}-K(b) d_{13} v_{4}\right], \\
& V_{3}=\frac{1}{d_{13}}\left[-K(a) a_{1} v_{1}-K(a) a_{3} v_{3}\right], \\
& V_{4}=\frac{1}{d_{13}}\left[K(a) b_{1} v_{1}+K(a) b_{3} v_{3}\right] .
\end{aligned}
$$

Here again the first two expressions equated to zero give us the adjoint boundary conditions, from which it is readily inferred that (19) is a necessary and sufficient condition that the given system be self-adjoint. The expressions for $V_{3}, V_{4}$, on the other hand, show us that the condition of Theorem II reduces to the fifth of the relations (18).

We might proceed in a precisely similar way in the four other cases. This, however, is not necessary, for we may get the desired results by a mere interchange of letters and subscripts in those already obtained. Thus, to deduce the result in the case $d_{42} \neq 0$ from that last obtained when we assumed $d_{13} \neq 0$, we notice that the bilinear form (20) remains unchanged if we interchange the subscripts 1 with 2 , and 3 with 4 , and at the same time interchange $K(a)$ and $-K(b)$. Since these interchanges leave equation (19) unchanged, and carry over the fifth equation (18) into the sixth, our statement for the case $d_{24} \neq 0$ is established.*

Similarly, from what we have proved in the case $d_{12} \neq 0$, we can get the facts just stated for the case $d_{23} \neq 0$ by interchanging the subscripts 1 and 3 (leaving 2 and 4 unchanged) and changing $K(a)$ into $-K(a)$, these changes leaving the form (20) and the equation (19) unchanged, and carrying over the first equation (18) into the third. In a similar way we interchange the subscripts 2 and 4 and change the sign of $K(b)$ in order to get the results for the case $d_{14} \neq 0$ from those for $d_{12} \neq 0$; and also to get the results when $d_{43} \neq 0$ from those when $d_{23} \neq 0$.

The case treated by Mason is the self-adjoint case, in which (19) is satisfied. $\dagger$ Here, on account of the relation

$$
d_{12} d_{34}+d_{13} d_{42}+d_{14} d_{23}=0,
$$

we see that, if the $d_{i j}$ 's in the first four relations (18) are all zero, the product $d_{13} d_{42}$ is also zero, and hence, by (19), all the $\mathrm{d}_{i j}$ 's are zero, contrary to hypothesis. Consequently in the self-adjoint case the condition of Theorem II may always be written in the form of at least one of the first four equations (18).

*These substitutions also carry over the expressions found above for $V_{1}, V_{2}, V_{3}, V_{4}$ into new forms which may be used if $d_{12} \neq 0$. In doing this, the subscripts of the $V$ 's should be left unchanged. A similar remark applies to the next paragraph.

$\dagger$ Mason himself used the term self-adjoint here, guided by a somewhat vague sense of analogy, but without having in mind the conception of an adjoint system in other cases. 
Without at first restricting ourselves to the self-adjoint case, let us suppose the reduced system has two-fold compatibility. Then every solution of the homogeneous equation $L(u)=0$ satisfies the conditions:

$$
\begin{aligned}
& a_{1} u(a)+a_{2} u(b)+a_{3} u^{\prime}(a)+a_{4} u^{\prime}(b)=0, \\
& b_{1} u(a)+b_{2} u(b)+b_{3} u^{\prime}(a)+b_{4} u^{\prime}(b)=0 .
\end{aligned}
$$

From this we can easily infer that $d_{13}$ and $d_{42}$ cannot both be zero; for if they were, eliminating first $u^{\prime}(b)$ and then $u(b)$ between the last written equations would give

$$
\begin{aligned}
& d_{14} u(a)+d_{34} u^{\prime}(a)=0, \\
& d_{12} u(a)+d_{32} u^{\prime}(a)=0,
\end{aligned}
$$

and since these equations are to hold for all solutions $u$ of the differential equation, for instance when $u(a)=1, u^{\prime}(a)=0$, and also when $u(a)=0$, $u^{\prime}(a)=1$, we should have

$$
d_{12}=d_{43}=d_{23}=d_{14}=0 \text {. }
$$

But this would make all the $d_{i j}$ 's zero. Hence if the reduced system is doubly compatible, the condition of Theorem II may always be written in the form of at least one of the last two equations (18).

In the self-adjoint case, neither of the quantities $d_{13}, d_{42}$ can be zero if the reduced system has two-fold compatibility, since by (19) they would both be zero, and this we have just seen is impossible. If the reduced system is selfadjoint and doubly compatible, the condition of Theorem II can always be written in both of the last two forms given in (18).

The results here stated, so far as they refer to the self-adjoint case, are essentially Mason's, though the statement here given is a trifle more complete than his.

\section{§3. Systems of Differential Equations.}

The extension to systems of linear differential equations is simple and fairly obvious, and will be here only briefly indicated.

Consider the differential expressions

$$
L_{i j}(u) \equiv l_{i j}^{[n]} \frac{d^{n} u}{d x^{n}}+l_{i j}^{[n-1]} \frac{d^{n-1} u}{d x^{n-1}}+\cdots+l_{i j}^{[0]} u \quad\left(\begin{array}{l}
i=1,2, \cdots m \\
j=1,2, \cdots m
\end{array}\right)
$$

and their adjoints $M_{i j}(v)$. We assume that the coefficients of $L_{i j}$ are not merely continuous throughout $S$, but are such that these adjoints exist and 
have continuous coefficients. * We have then Lagrange's identity

$$
v L_{i j}(u)-u M_{i j}(v)=\frac{d}{d x} P_{i j}(u, v) .
$$

Now consider the $m$ homogeneous linear differential expressions of the $m$ th order with the $m$ dependent variables $u_{1}, \cdots u_{m}$ :

$$
L_{i}\left(u_{1}, \cdots u_{m}\right) \equiv \sum_{j=1}^{m} L_{i j}\left(u_{j}\right) \quad(i=1,2, \cdots m)
$$

By the adjoint of this system of expressions we understand the system

$$
M_{i}\left(v_{1}, \cdots v_{m}\right) \equiv \sum_{j=1}^{m} M_{j i}\left(v_{j}\right) \quad(i=1,2, \cdots m)
$$

By replacing $u$ by $u_{j}$ and $v$ by $v_{i}$ in (21) and summing with regard to $i$ and $j$, we find

$$
\sum_{i=1}^{m}\left[v_{i} L_{i}\left(u_{1}, \cdots u_{m}\right)-u_{i} M_{i}\left(v_{1}, \cdots v_{m}\right)\right]=\frac{d}{d x} \sum_{i=1}^{m} \sum_{j=1}^{m} P_{j_{l}}\left(u_{i}, v_{j}\right) .
$$

This we call Lagrange's identity for the system (22). The second member is the derivative of a bilinear form in $u_{i}, u_{i}^{\prime}, \cdots u_{i}^{[n-1]}, v_{i}, v_{i}^{\prime}, \cdots v_{i}^{[n-1]}$ $(i=1,2, \cdots m)$, whose determinant (cf. the second footnote to $\S 1$ ) is, except perhaps for sign, the $n$th power of the determinant $\dagger$

$$
\Delta=\left|\begin{array}{lll}
l_{11}^{[n]} & \ldots & l_{1 m}^{[n]} \\
\ldots & \ldots & \ldots \\
\ldots & \ldots & \ldots \\
l_{m 1}^{[n]} & \ldots & l_{m m}^{[n]}
\end{array}\right| .
$$

We shall assume that $\Delta$ does not ranish at any point of $S$. Then integrating (24) from $a$ to $b$, we get Green's theorem, in which the second member is a non-singular bilinear form in

$$
\begin{array}{llll}
u_{i}(a), & \cdots u_{i}^{[n-1]}(a), & u_{i}(b), & \cdots u_{i}^{[n-1]}(b) \\
v_{i}(a), & \cdots v_{i}^{[n-1]}(a), & v_{i}(b), & \cdots v_{i}^{[n-1]}(b) \quad(i=1, \cdots m) .
\end{array}
$$

Let us now denote by $U_{1}\left(u_{1}, \cdots u_{m}\right), \cdots U_{2 n m}\left(u_{1}, \cdots u_{m}\right)$ linearly independent linear forms in (25). We can then write Green's theorem in

* Cf. the first footnote to $\$ 1$. See also the closing lines of the present section.

$\dagger$ The proof of this statement requires a little manipulation of determinants, which, however, presents no difficulty. 
the form

$$
\begin{aligned}
\int_{a}^{b} \sum_{i=1}^{m}\left[v_{i} L_{i}\left(u_{1}, \cdots u_{m}\right)-u_{i} M_{i}\left(v_{1}, \cdots v_{m}\right)\right] d x=U_{1} V_{2 n m} & +\cdots \\
& +U_{2 n m} V_{1},
\end{aligned}
$$

where the $V_{i}$ 's are linearly independent linear forms in (26).

Now consider the complete system

the reduced system

$$
\begin{array}{lc}
L_{i}\left(u_{1}, \cdots u_{m}\right)=r_{i} & (i=1, \cdots m), \\
U_{i}\left(u_{1}, \cdots u_{m}\right)=\gamma_{i} & (i=1, \cdots n m),
\end{array}
$$

and the adjoint system

$$
\begin{array}{lc}
L_{i}\left(u_{1}, \cdots u_{m}\right)=0 & (i=1, \cdots m) \\
U_{i}\left(u, \cdots u_{m}\right)=0 & (i=1, \cdots n m)
\end{array}
$$

$$
\begin{array}{lc}
M_{i}\left(v_{1}, \cdots v_{m}\right)=0 & (i=1, \cdots m) \\
V_{i}\left(v_{1}, \cdots v_{m}\right)=0 & (i=1, \cdots n m) .
\end{array}
$$

The condition we have imposed, that $\Delta$ does not vanish in $S$, means precisely that the systems of differential equations in (28), (29), and (30) have no singular points in $S$. Consequently there exists one and only one system of functions which satisfy the differential equations of any one of these systems and at a point of $S$ take on, along with their first $n-1$ derivatives, arbitrarily given values.

By the order of compatibility of the system (29) we understand the number of linearly independent systems of functions $u_{1}, \cdots u_{m}$ which satisfy it. When we say that the system (29) is incompatible, or has zero-fold compatibility, we mean that the only systems of functions satisfying (29) are those which are all identically zero.

With these preliminaries, the extension of the methods and results of $\$ \$ 1,2$ to the case we are now considering presents no difficulty. We content ourselves, therefore, with merely stating the main results, which correspond to Theorems I and II.

Theorem III. If the homogeneous system (29) has $k$-fold compatibility, the adjoint system (30) also has $k$-fold compatibility.

Theorem IV. A necessary and sufficient condition that the complete system (28) have a solution is that every solution $\left(v_{1}, \cdots v_{m}\right)$ of the homogeneous adjoint system (30) satisfy the relation

$\int_{a}^{b}\left[v_{1} r_{1}+\cdots+v_{m} r_{m}\right] d x=\gamma_{1} V_{2 n m}\left(v_{1}, \cdots v_{m}^{\prime}\right)+\cdots+\gamma_{n m} V_{n m+1}\left(v_{1}, \cdots v_{m}\right)$ 
The case $n=1$, in which the differential equations of the system are of the first order, is peculiarly important since, as is well known, all other cases may, if we wish, be reduced to this. We may here solve the equations for the derivatives, as we may always solve for the derivatives of highest order, and thus write

$$
\begin{aligned}
& L_{i}\left(u_{1}, \cdots u_{m}\right) \equiv \frac{d u_{i}}{d x}+a_{i 1}(x) u_{1}+\cdots+a_{i m}(x) u_{m} \\
& M_{i}\left(v_{1}, \cdots v_{m}\right) \equiv-\frac{d v_{i}}{d x}+a_{1 i}(x) v_{1}+\cdots+a_{m i}(x) v_{m}
\end{aligned}
$$

and Green's Theorem becomes

$$
\begin{aligned}
\int_{a}^{b} \sum_{i=1}^{m}[ & \left.v_{i} L_{i}\left(u_{1}, \cdots u_{m}\right)-u_{i} M_{i}\left(v_{1}, \cdots v_{m}\right)\right] d x=-u_{1}(a) v_{1}(a) \\
& -\cdots-u_{m}(a) v_{m}(a)+u_{1}(b) v_{1}(b)+\cdots+u_{m}(b) v_{m}(b) .
\end{aligned}
$$

Consequently if

$$
\begin{array}{r}
U_{i}\left(u_{1}, \cdots u_{m}\right) \equiv \alpha_{i 1} u_{1}(a)+\cdots+\alpha_{i m} u_{m}(a)+\beta_{i 1} u_{1}(b)+\cdots+\beta_{i m} u_{m}(b) \\
(i=1, \cdots 2 m)
\end{array}
$$

where the determinant $D$ formed from the $\alpha$ 's and $\beta$ 's is not zero, we shall have

$$
\begin{aligned}
V_{2 m+1-i}\left(v_{1}, \cdots v_{m}\right) \equiv \frac{1}{D}\left[-A_{i 1} v_{1}(a)-\right. & \cdots-A_{i m} v_{m}(a) \\
& \left.+B_{i 1} v_{1}(b)+\cdots+B_{i m} v_{m}(b)\right]
\end{aligned}
$$

where $A_{i j}$ and $B_{i j}$ are the cofactors of $\alpha_{i j}$ and $\beta_{i j}$ in the determinant $D$.

We now see that this case $n=1$ is peculiarly simple in the following respect*: In the case of a system of equations of the first order written in the form (31) the adjoint conditions $V_{i}=0$ depend merely, and in a simple and explicit rational manner, on the original conditions $U_{i}=0$, whereas in general, even in the case of a single differential equation of order higher than the first, they depend also on the coefficients of the differential equations.

Another peculiarity worthy of remark in the case $n=1$, when the form (31) is used, is that no demand on the coefficients of the differential equations need be made beyond their mere continuity in $S$, since the derivatives of these coefficients nowhere occur in our work, so that even the existence of their derivatives need not be demanded.

* This simple case has been considered by Bounitzky in Liouville's $\mathrm{J}$ ou r nal, 6th series, vol. 5 (1909), p. 65, who, however, does not obtain the special case $n=1$ of Theorems III and IV, being concerned with a quite different class of applications of adjoint systems. 
By the aid of this last remark it is easy to see how all demands concerning the existence or continuity of derivatives of the coefficients of the differential equations may be avoided even when the order of these equations is greater than one: we have merely to replace these equations by systems of equations of the first order in the form (31) and apply Theorem IV to these systems. A little consideration, however, will show how we can accomplish the same object without reduction to a system of equations of the first order and can even generalize a step farther. For the sake of simplicity, I will explain this for the typical case of a single equation of the third order.

Let

$$
\begin{aligned}
L(u) \equiv a_{3} \frac{d}{d x}\left\{a_{2} \frac{d}{d x}\right. & {\left.\left[a_{1} \frac{d}{d x}\left(a_{0} u\right)+b_{1} u\right]+b_{2} u\right\} } \\
& +l_{2} \frac{d}{d x}\left[a_{1} \frac{d}{d x}\left(a_{0} u\right)+b_{1} u\right]+l_{1} \frac{d}{d x}\left(a_{0} u\right)+q u,
\end{aligned}
$$

where the $a^{\prime} \mathrm{s}, b$ 's, $l$ 's, and $q$ are functions of $x$ continuous throughout $S$. If these functions had continuous derivatives of certain orders, this would reduce at once to the form of expression considered in $\S 1$. This will, in particular, always be the case if we are dealing with analytic functions. We wish, however, now to consider the case in which the $a$ 's, $b$ 's, $l$ 's, and $q$ are continuous functions of the real variable $x$ concerning which we make no assumption whatever as to the existence of derivatives. The expression $L(u)$ then includes the expressions of $\S 1$ as very special cases.

The theory of the equation*

$$
L(u)=r,
$$

where $r$ is also continuous in $S$, and where we assume that none of the functions $a_{0}, a_{1}, a_{2}, a_{3}$ vanishes in $S$, may be readily obtained by letting

$$
y_{1}=a_{0} u, \quad y_{2}=b_{1} u+a_{1} y_{1}^{\prime}, \quad y_{3}=b_{2} u+a_{2} y_{2}^{\prime} .
$$

These functions, $y_{1}, y_{2}, y_{3}$, are then seen to satisfy a system of linear differential equations of the first order with no singular point in $S$, and we thus

* It should be noticed that this is not a differential equation in the ordinary sense since it is not a relation between $u, u^{\prime}, u^{\prime \prime}, u^{\prime \prime \prime}$. Indeed a solution of this equation will not in general possess even a first derivative. We shall, however, use the term linear differential equation in an extended sense to cover such equations as this. Their theory might readily be developed on an independent basis (instead of by the reduction to a system of the first order as indicated in the text), the method of successive approximations, for instance, being available to establish the existence theorem. The only mention of such equations which I have seen is the very special one by WEYL referred to at the close of this paper. 
infer that the equation (34) has one and only one solution $u$ for which

$$
u, \quad \frac{d}{d x}\left(a_{0} u\right), \quad \frac{d}{d x}\left[a_{1} \frac{d}{d x}\left(a_{0} u\right)+b_{1} u\right]
$$

take on arbitrarily given values at an arbitrarily given point of $S$. Moreover the general solution of (34) is readily seen to be obtained by adding to any particular solution the general solution of the reduced equation

$$
L(u)=0 \text {. }
$$

Finally, the solutions of this homogeneous equation are seen to form a ternary family, so that we may speak of any three linearly independent solutions as forming a fundamental system. In short, all the essential facts concerning linear differential equations apply without change to this case, with the exception of the change just noted in the existence theorem.

By the expression $M(v)$ adjoint to $L(u)$ we shall understand

$$
\begin{aligned}
& M(v)=-a_{0} \frac{d}{d x}\left\{a_{1} \frac{d}{d x}\left[a_{2} \frac{d}{d x}\left(a_{3} v\right)-l_{2} v\right]+l_{1} v\right\} \\
& +b_{1} \frac{d}{d x}\left[a_{2} \frac{d}{d x}\left(a_{3} v\right)-l_{2} v\right]-b_{2} \frac{d}{d x}\left(a_{3} v\right)+q v .
\end{aligned}
$$

Between these adjoint expressions there is a Lagrange identity of precisely the form given in $\S 1$, where, however, $P(u, v)$ now stands for a non-singular bilinear form not in $u, u^{\prime}, u^{\prime \prime}, v, v^{\prime}, v^{\prime \prime}$, but in the three quantities (35), as the first set of variables, and the three quantities

$$
v, \quad \frac{d}{d x}\left(a_{3} v\right), \quad \frac{d}{d x}\left[a_{2} \frac{d}{d x}\left(a_{3} v\right)-l_{2} v\right]
$$

as the second set. If we take for the $U$ 's linearly independent linear forms in the six values that (35) take on at $a$ and $b$ respectively, the $V$ 's, when we write Green's theorem in the form (3), will be linearly independent linear forms in the six values which the quantities (38) take on at the points $a$ and $b$. With this understanding all the work in $\S 1$ is applicable if $L$ and $M$ have the more general definitions (33) and (37), and Theorems I and II hold without further change for this more general case.* In particular, $L$ may have the

* We might consider the even more general form of equation

$$
\begin{aligned}
a_{3} \frac{d}{d x}\left\{a_{2} \frac{d}{d x}\left[a_{1}, \frac{d}{d x}\left(a_{0} u-r_{0}\right)+b_{1} u-r_{1}\right]+b_{2} u-r_{2}\right\} \\
\quad+l_{2} \frac{d}{d x}\left[a_{1} \frac{d}{d x}\left(a_{0} u-r_{0}\right)+b_{1} u-r_{1}\right]+l_{1} \frac{d}{d x}\left(a_{0} u-r_{0}\right)+q u=r .
\end{aligned}
$$


form of $\S 1$ where no restriction as to the differentiability of the coefficients is imposed. It should be noticed, however, that in this case $M$ will no longer have the form of $\S 1$, but another special case of the general form of this section.

The case in which $L$ has the form (16), $K$ being merely assumed continuous, is a very special case of what we have just been discussing. The fact that the existence of the derivative of $K$ need not here be demanded has been mentioned by WEYL.*

\section{$\S 4$. An Extension of the Conception of Adjoint System.}

Let us now consider the system

$$
\begin{aligned}
L(u) & =r \\
U_{i}(u) & =\gamma_{i} \quad(i=1,2, \cdots m),
\end{aligned}
$$

where, instead of $n$ boundary conditions, we have $m$. We shall assume these boundary conditions to be linearly independent, so that $m \overline{<} 2 n$. The expression $L(u)$ may be taken either in the restricted sense of $\$ 1$ or in the broader sense explained at the end of $\S 3$. By the side of this complete system, we have the reduced system

$$
\begin{aligned}
L(u) & =0 \\
U_{i}(u) & =0 \quad(i=1,2, \cdots m) .
\end{aligned}
$$

We now form $2 n-m$ further linear forms $U_{m+1}(u), \cdots U_{2 n}(u)$ so that the functions $U_{1}, \cdots U_{2 n}$ are linearly independent. From these, as in $\S 1$, we form the expressions $V_{1}, \cdots V_{2 n}$, which will also be linearly independent, such that Green's theorem may be written in the form (3). Then the system

$$
\begin{aligned}
& M(v)=0 \\
& V_{i}(v)=0 \quad(i=1,2, \cdots 2 n-m)
\end{aligned}
$$

we call the system adjoint to $\left(6^{\prime}\right),\left(7^{\prime}\right)$.

This can readily be reduced to a system of linear differential equations of the first order, and we thus see that, apart from a certain obvious change in the existence theorem, the theory of this equation does not differ substantially from that of the ordinary non-homogeneous equation so far as the more fundamental theorems go. Nevertheless, since this equation cannot be written $L(u)=f(x)$, where $L$ is homogeneous, the reasoning used to establish Theorem II is no longer applicable, and it is readily seen that this theorem must be replaced by a more complex one similar to (and of course a special case of) Theorem IV. The formula involved contains the integral of a sum of products, not that of a single product.

* Mathematische Annalen, vol. 68 (1910), p. 221. 
If the three systems of $\S 1$ are replaced by these three, Lemmas I, II, IV of that section hold with only the obvious modification that the matrices in Lemmas I and II now have $m$ rows instead of $n$, while in Lemma IV the subscripts of the $U$ 's must begin with $m+1$. The proofs remain essentially unchanged.

Lemma III must be replaced by the following:

LEMmA III'. When $m \leqq n$, the complete system $\left(4^{\prime}\right),\left(5^{\prime}\right)$ always has a solution if the reduced system has $(n-m)$-fold compatibility.

The proof is substantially that of Lemma III.

A modification is also necessary in the statement of Theorem $I$.

Theorem I'. If a homogeneous system which involves $m$ linearly independent boundary conditions, and where the differential equation is of order $n$, has $k$-fold compatibility, then $k+m-n \geqq 0$, and this number is precisely the order of compatibility of the adjoint system $\left(8^{\prime}\right),\left(9^{\prime}\right)$.

The proof follows closely that given above for Theorem I. We show first that, if $\kappa$ is the order of compatibility of $\left(8^{\prime}\right),\left(9^{\prime}\right), \kappa \bar{\Sigma} k+m-n$. Consequently, since $\left(6^{\prime}\right),\left(7^{\prime}\right)$ is the adjoint of $\left(8^{\prime}\right),\left(9^{\prime}\right)$, we must also have $k \equiv \kappa+(2 n-m)-n$; that is $\kappa \bar{\Sigma} k+m-n$. From a comparison of these two inequalities our theorem follows.

Theorem II'. A necessary and sufficient condition that the complete system $\left(4^{\prime}\right),\left(5^{\prime}\right)$ have a solution is that every solution, $v$, of the homogeneous adjoint system $\left(8^{\prime}\right),\left(9^{\prime}\right)$ satisfy the relation

$$
\int_{a}^{b} v r d x=\gamma_{1} V_{2 n}(v)+\cdots+\gamma_{m} V_{2 n-m+1}(v)
$$

Here again the proof is substantially that of Theorem II, the case $n \bar{\Sigma}$, $k=n-m$ being first disposed of by means of Lemma III'.

This theorem of course contains Theorem II, and hence Mason's results, as a special case. The only other special case $I$ have found in the literature is that in which $m=n+1$ ( $n$ arbitrary), $U_{1}(u)=u(a), U_{2}(u)=u^{\prime}(a)$, $\cdots U_{n}(u)=u^{[n-1]}(a), U_{n+1}(u)=\beta u(b)+\beta^{\prime} u^{\prime}(b)+\cdots+\beta^{[n-1]} u^{[n-1]}(b)$; while $\gamma_{1}=\cdots=\gamma_{n}=0$. In this case, $k=0, \kappa=1$. There is therefore essentially only one solution, $v$, of the adjoint system, and the condition that the complete system have a solution is

$$
\int_{a}^{b} v r d x=0
$$

A condition of exactly this form is obtained by DinI* by quite another

*Annali di Matematica, vol. 12 (1906), p. 226.

Trans. Am. Math. Soc. 28 
method, and it is not obvious at first sight that his function $v$ (called in his notation $\left.D_{1 n} / a_{0} D\right)$ is the same as the one our method gives. This is, however, actually the case, as may be easily proved, and consequently this result of Dini may be regarded as a very special case, stated in a somewhat different form, of Theorem II'.

The possibility of extending the results of this section to systems of differential equations is obvious.

HaRVARd University,

Cambridge, Mass. 